\title{
The role of the WWOX gene in leukemia and its mechanisms of action
}

\author{
ZHAOLEI CUI, DONGHONG LIN, FENG CHENG, LINGQING LUO, \\ LINGYING KONG, JIANPING XU, JIANDA HU and FENGHUA LAN
}

\begin{abstract}
Department of Clinical Laboratory, School of Medical Technology and Engineering, Fujian Medical University, Fuzhou, Fujian 350004, P.R. China
\end{abstract}

Received December 7, 2012; Accepted February 27, 2013

DOI: $10.3892 / o r .2013 .2361$

\begin{abstract}
The WW domain-containing oxidoreductase (WWOX) gene which encompasses the common human fragile site FRA16D has been proposed as a putative tumor suppressor gene, and loss of WWOX expression has been found in several types of solid cancer. As the role of WWOX in human leukemia has not yet been fully elucidated, the present study examined the expression of WWOX in patients with different types of leukemia as well as in leukemia-derived cell lines. Based on the data, WWOX mRNA (WWOX) and protein (Wwox) were significantly reduced or absent in the leukemia patients as well as in the cell lines. In addition, a recombinant expression vector, pGC-FU-WWOX, was constructed and transfected WWOX cDNA into Jurkat cells (acute T-lymphoblastic leukemia) and K562 cells (chronic myeloid leukemia in erythroid crisis) which all lack endogenous Wwox. In vitro experiments indicated that restoration of Wwox in Jurkat and K562 cells significantly suppressed proliferation and colony formation. Of note, apoptosis was also induced by Wwox restoration. Furthermore, we traced the mechanisms underlying this process and found that Wwox restoration could trigger the mitochondrial pathway in leukemia. Our data provide evidence that WWOX exerts a role as a tumor suppressor gene in leukemia, possibly by inhibiting proliferation and promoting apoptosis via the mitochondrial pathway.
\end{abstract}

\section{Introduction}

The WW domain-containing oxidoreductase (WWOX) gene is located at a chromosome region, 16q23.3-24.1, that spans the second most common human fragile site, FRA16D (1). The WWOX gene has nine exons and encodes a Wwox protein with a molecular weight for $46 \mathrm{kDa}$. The Wwox protein contains

Correspondence to: Professor Donghong Lin, Department of Clinical Laboratory, School of Medical Technology and Engineering, Fujian Medical University, 88 Jiaotong Road, Taijiang, Fuzhou, Fujian 350004, P.R. China

E-mail: lindh65@163.com; cuileidizi@163.com

Key words: WW domain-containing oxidoreductase, restoration, apoptosis, leukemia, mechanism, mitochondria two N-terminal WW domains and one C-terminal short-chain dehydrogenase domain known as $\operatorname{SDR}(1,2)$. WW domains are characterized by their interactions with proline-containing ligands, which is a basis for protein-protein interactions (3), while the SDR domain is located in the central region of the Wwox protein and is shared by steroid-hormone-metabolizing enzymes and characterized by its high frequency loss or alteration implicated in numerous types of carcinomas $(3,4)$.

To date, numerous studies have shown either a reduction or loss of WWOX expression in a variety of carcinomas, including breast, esophagus, pancreas and thyroid cancer (5-9). Significantly, a restoration or upregulation of WWOX in tumors such as lung and breast cancer can sensitize them to apoptosis both in vitro and in vivo $(10,11)$. Studies of WWOX animal models suggest that an inactivation of one allele for WWOX accelerates the predisposition of normal cells to malignant transformation (12). In addition, WWOX knockout mice also showed a shortened life span and defects in bone metabolism, as well as other deficiencies $(11,13)$. These studies indicate that WWOX may function as a tumor suppressor gene.

All the results cited above verified the functional role of WWOX or its protein product in tumorigenesis in leukemia, particularly aberration or absence of WWOX expression in primary hematopoietic malignancies has also been reported $(14,15)$. This prompted us to hypothesize that WWOX may function as a tumor suppressor in leukemia, also due to its special location on FRA16D which is susceptible to activation by carcinogens. Studies on the biological role of WWOX in tumorigenesis have shown that its function in tumor cellular metabolism is likely to modulate cell viability by interacting with factors involved in cell apoptosis control such as Bcl-2, Bcl-xL and caspase (16-19), which suggests a close relationship between WWOX and the apoptosis signal pathways. However, this relationship remains to be confirmed.

In the present study, we first evaluated the expression of WWOX in 38 cases of primary leukemia patients and 10 leukemia cell lines. With a reduced or absent expression of WWOX observed in our results, we restored Wwox expression in its negative cell lines as Jurkat and K562 cells by transfecting them with pGC-FU-WWOX lentiviral plasmid, and we observed that the Jurkat and K562 cells all underwent viability inhibition and apoptosis following the restoration in vitro. To elucidate the underlying mechanisms, we further examined 
whether WWOX regulates cell death via the mitochondrial pathway, and we found an activation of the mitochondrial pathway in WWOX-mediated apoptosis in human leukemia.

\section{Materials and methods}

Samples, cell lines and cell culture. Thirty-eight diagnosed leukemia patients at Fujian Medical University Affiliated Xiehe Hospital from September 2010 to June 2011 were enrolled in the study. These patients included 9 cases of acute lymphoblastic leukemia (ALL), 18 cases of acute myelogenous leukemia (AML) and 11 cases of chronic myelogenous leukemia (CML). Mononuclear cells purified from 20 healthy volunteers were used as controls. Human Jurkat (acute T-lymphoblastic leukemia), K562 (chronic myelogenous leukemia in erythroid crisis), $\mathrm{NB}_{4}$ (promyelocytic leukemia), HL-60 (acute myelogenous leukemia), U937 (promonocytic leukemia), CEM (acute T-lymphocyte leukemia), U266 (human myeloma), KAR (K562 resistance to doxorubicin), ADR (HL-60 resistance to doxorubicin) and CA46 (human Burkitt's lymphoma) cell lines were all purchased from the cell bank of the Chinese Academy of Medical Sciences and maintained in RPMI-1640 supplemented with $10 \%$ fetal bovine serum (FBS) (Gibco, USA), and cultured at $37^{\circ} \mathrm{C}$ in a $5 \% \mathrm{CO}_{2}$ humidified atmosphere.

Reverse transcription-PCR analysis. Total RNA was extracted with TRIzol Reagent (Invitrogen, USA) and was reverse transcribed into cDNA using a commercial kit (Fermentas, USA). We then amplified 6-8 exons of WWOX with the primers (20). The primers of an internal control gene, glyceraldehyde 3-phosphate dehydrogenase (GAPDH), were: forward, 5'-CAAGGTCATCCATGACAACTTTG-3' and reverse, 5'-GTCCACCACCCTGTTGCTGTAG-3'. PCR was performed using a PCR kit (Biomed, Beijing, China) under the recommended conditions at an initial denaturation for $5 \mathrm{~min}$ at $94^{\circ} \mathrm{C}$ followed by 30 cycles of $94^{\circ} \mathrm{C}$ for $30 \mathrm{sec}, 55^{\circ} \mathrm{C}$ for $30 \mathrm{sec}, 72^{\circ} \mathrm{C}$ for $30 \mathrm{sec}$, and a final extension for $7 \mathrm{~min}$ at $72^{\circ} \mathrm{C}$. Quantified data were normalized to GAPDH.

In vitro lentiviral transduction and growth condition observation. The pGC-FU-WWOX lentiviral vector was constructed by our laboratory, amplified and titrated based on the manufacturer's instructions (Genechem, Shanghai, China) (21). A pGC-FU-GFP vector served as negative control (mock lentiviral vector only encoding GFP). Cells were seeded at a density of $1 \times 10^{5} / \mathrm{ml}$ in 6 -well plates and infected with pGCFU-WWOX at appropriate multiplicities of infection (MOI). The transduction efficiency and growth conditions were evaluated by visualization of green fluorescent protein (Wwox-GFP fusion protein) and cell morphological features every $24 \mathrm{~h}$ following infection using a fluorescence microscope (Nikon, Tokyo, Japan).

Proliferation assay. Proliferation inhibition ratio was assessed by CCK- 8 assay (Dojindo, Japan). Briefly, $5 \times 10^{3}$ cells/well were seeded with a total volume of $100 \mu 1$ in 96 -well plates. Cell infection followed the procedures above. Every $24 \mathrm{~h}$ following incubation, $10 \mu \mathrm{l}$ CCK- 8 was added to each well followed by incubation for another $3 \mathrm{~h}$ at $37^{\circ} \mathrm{C}$ in a $5 \% \mathrm{CO}_{2}$ humidified atmosphere. The optical density (OD) value was measured by a microculture plate reader (BioTek Instruments, USA) using double-wavelength (450 and $630 \mathrm{~nm}$ ). Growth inhibition ratio $(\%)=(1-$ Mean OD value of lentivirus infected cells/Mean OD value of non-treated cells) x 100\%. Each assay was performed in triplicate.

Colony-forming assay. Briefly, GFP-expressing cells were sorted by flow cytometry using Vi-Cell counter (Beckman Coulter, Fullerton, CA, USA), and 300 cells $/ \mathrm{ml}$ were seeded in 24-well plates. Methylcellulose dissolved in RPMI-1640 containing $30 \%$ FBS was added to each well with a concentration of $0.8 \mathrm{~g} / \mathrm{l}$. The colonies containing $>50$ cells were counted on Day 14. Each assay was performed in triplicate.

DAPI fluorescence staining and DNA fragmentation analysis. For DAPI fluorescence staining, cells were collected and washed 2 times with PBS and stained by DAPI (Beyotime, Shanghai, China) in a concentration of $2 \mathrm{mg} / \mathrm{ml}$ for 3-5 min, then washed 2 times with PBS. Morphological changes of the stained cells were examined using the fluorescence microscope. DNA fragmentation analysis was performed according to the protocols of a commercial DNA Fragmentation Kit (Beyotime), followed by agarose gel electrophoresis: each sample containing equal amounts of extracted DNA $(2 \mu \mathrm{g})$ in $1.0 \%$ agarose gel with a constant voltage $18 \mathrm{~V}$ for $4-6 \mathrm{~h}$.

Immunofluorescence staining. In brief, cell monolayers were fixed with $4 \%$ paraformaldehyde, and incubated at $4^{\circ} \mathrm{C}$ overnight with rabbit anti-human Wwox, 1:500, and mouse monoclonal anti-human cytochrome $c, 1: 500$ (Abcam, USA). FITC-conjugated goat anti-rabbit and Cy3-conjugated goat anti-mouse IgG (Beyotime) were all diluted in 1:1,000. DAPI was used to stain the nucleus. Stained cell monolayers were washed 2 times with PBS and observed under the fluorescence microscope.

Real-time PCR analysis. Real-time PCR was enabled using a SYBR-Green PCR Master Mix (Roche Diagnostics GmbH, Germany) under the recommended conditions. The primer sequences for Bcl-2 and Bax were described previously (22), and for GAPDH they were: forward, 5'-GAAGGTGAAG GTCGGAGT-3' and reverse, 5'-GAAGATGGTGATGGGA TTTC-3'. The comparative Ct method to GAPDH was used to calculate the relative expression level of Bcl-2 and Bax.

Western blot analysis. A Cell Mitochondria Isolation Kit (Beyotime) was used in the isolation of proteins from the nucleus, cytoplasm and mitochondria of the cells. The primary antibodies and the dilutions were: rabbit anti-human Wwox, 1:1,000; anti-Bcl-2 (1:500; Abcam, USA); anti-caspase-9 (1:300; Santa Cruz, Biotechnology, Inc., Santa Cruz, CA, USA); anti-caspase-3 (1:500; Beyotime). Mouse monoclonal anti-human $\beta$-actin and anti-tubulin (Beyotime) were all diluted in 1:1,000 and used as controls.

Statistical analysis. Data are expressed as the means \pm standard deviation (SD), and analyzed by Student's t-test, or non-parametric test using the SPSS 13.0 (SPSS Inc., Chicago, IL, USA). $\mathrm{P}<0.05$ was considered to indicate a statistically significant difference. 
A ALL

AML

CML

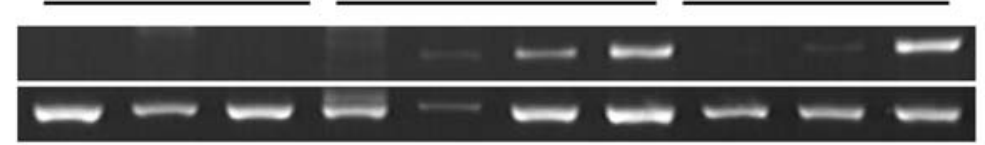

WWOX

GAPDH

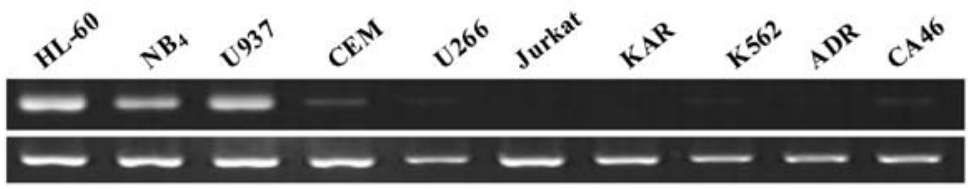

WWOX

GAPDH

Healthy volunteers

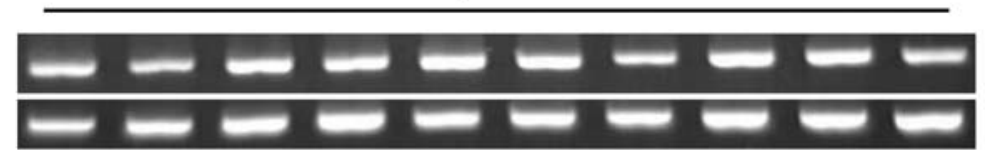

WWOX

GAPDH

B
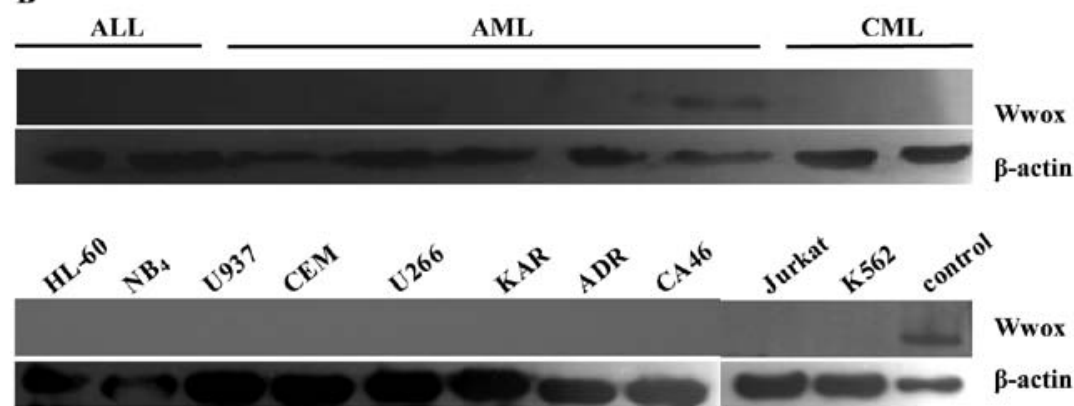

Healthy volunteers

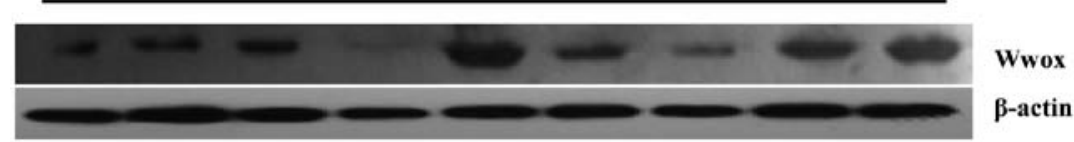

Figure 1. Expression of WWOX in leukemia patients and cell lines. The expression level of WWOX mRNA and Wwox protein in leukemia patients and cell lines was assessed by RT-PCR and western blot analysis. (A) RT-PCR analysis. RNA was extracted from mononuclear cells purified from 38 leukemia patients, and 20 healthy volunteers were used as controls. (B) Western blot analysis. Protein was also extracted from mononuclear cells, and controls were the same as in RT-PCR.

\section{Results}

Expression of WWOX is reduced or absent in leukemia. We examined the expression level of WWOX mRNA and the Wwox protein in leukemia patients and cell lines via RT-PCR and western blot analysis. Twenty-eight of the 38 leukemia cases (74\%) showed reduced or lost expression of WWOX, and included 11 AML (39\%), 8 ALL (29\%) and 9 CML (32\%) patients (Fig. 1A). For Wwox, only 6/38 cases (16\%) had a positive expression (Fig. 1B). Similarly, 7/10 leukemic cell lines (70\%) showed absent or reduced WWOX expression; Jurkat and KAR cells were negative, while K562, CEM, U266, CA46 and ADR cells had a low expression level, and only HL-60, $\mathrm{NB}_{4}$ as well as U937 cells exhibited a high expression level similar to the normal controls (Fig. 1A). Endogenous Wwox in all leukemic cells was undetectable (Fig. 1B). All controls exhibited a high expression level of both WWOX mRNA and Wwox protein (Fig. 1).

Wwox restoration suppresses cell proliferation and colony formation in Jurkat and K562 cells. We first examined whether Wwox was successfully restored in Jurkat and K562 cells via fluorescence microscope and western blot analysis. The results showed that pGC-FU-WWOX- and pGC-FU-GFPinfected cells all expressed GFP at $24 \mathrm{~h}$ following infection (Fig. 2B). Cells infected with pGC-FU-WWOX exhibited an increased expression level of Wwox with time lapse (Fig. 2A), while pGC-FU-GFP-infected cells had no expression (data not shown), indicating that Wwox was successfully restored in the Jurkat and K562 cells. In addition, pGC-FU-WWOX-infected cells presented anti-proliferation morphological features characterized by cell shrinkage and colonies decreased with time lapse (Fig. 2B). Wwox restoration resulted in significantly reduced cell proliferation with the proliferation inhibition rate increasing from $9.1 \%$ at $48 \mathrm{~h}$ to $74.62 \%$ at $96 \mathrm{~h}$ for Jurkat cells, and from $3.23 \%$ at $48 \mathrm{~h}$ to $40.68 \%$ at $96 \mathrm{~h}$ for K562 cells, all with $\mathrm{P}<0.05$ when vs. pGC-FU-GFP-infected cells (Fig. 2C). Moreover, colony formation numbers of pGCFU-WWOX-infected cell lines were significantly lower than those of non-treated cells $(\mathrm{P}<0.01$, for both Jurkat and K562 cells), while pGC-FU-GFP-infected cells showed no statistical significance vs. non-treated cells with $\mathrm{P}>0.05$ (Fig. 2D). 
A
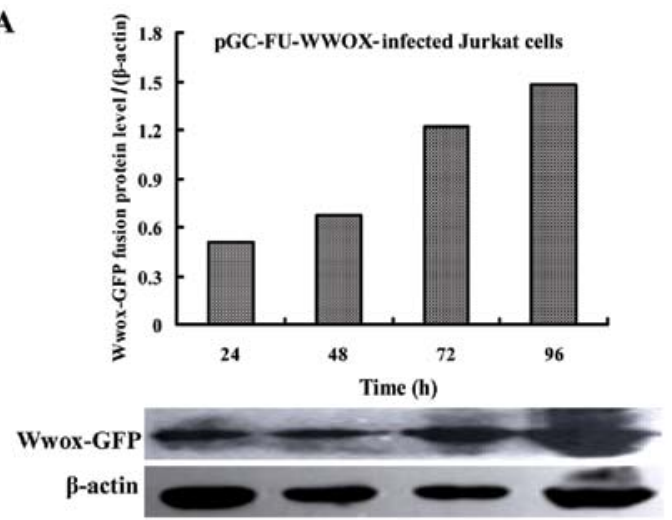

B
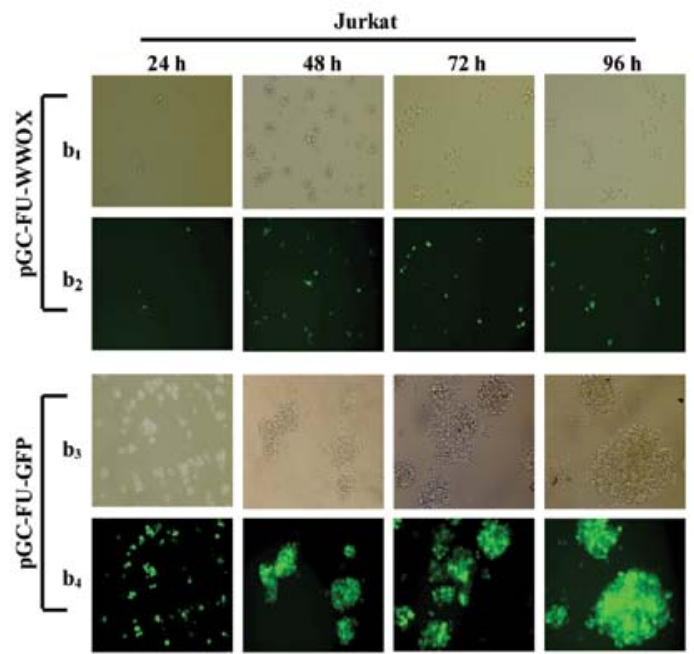

C
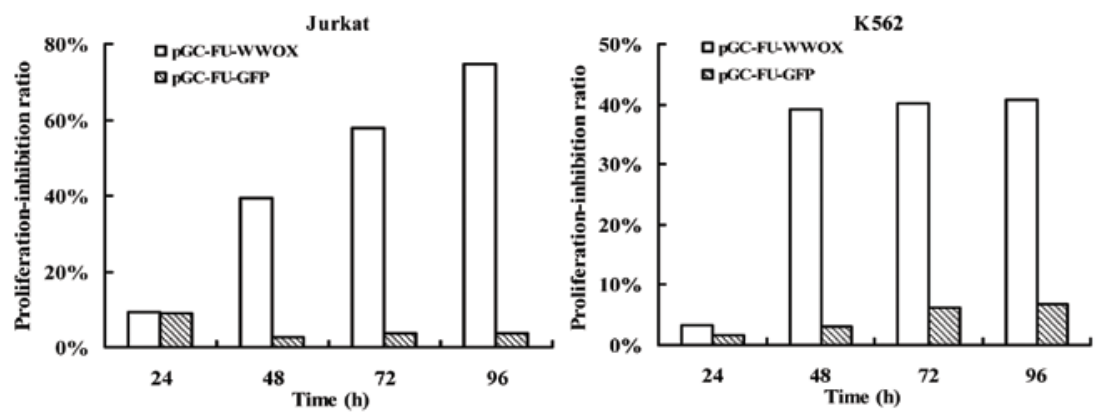

D
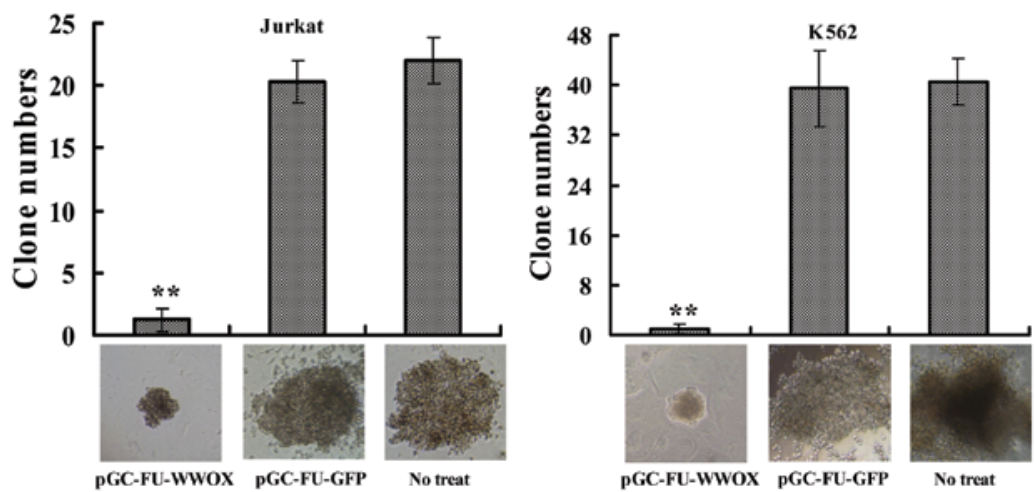

Figure 2. Effects of Wwox restoration on cell proliferation and colony formation on Jurkat and K562 cells. (A) Expression of Wwox-GFP fusion protein in pGC-FU-WWOX-infected Jurkat and K562 cells at different time points detected by western blot analysis. (B) Expression of GFP at different time points as well as cell morphological features observed by fluorescence microscope, x200 (b1 and b3, ordinary light; b2 and b4, fluorescent light). (C) The proliferation ratio for lentivirus-infected cells measured by CCK-8 assay. (D) Colony-forming assay. Cell colonies containing $>50$ cells were counted on Day 14 with a microscope, $x 200$. Data shown are the means $\pm S D(n=15) .{ }^{* *} \mathrm{P}<0.01$, vs. non-treated cells. 
A

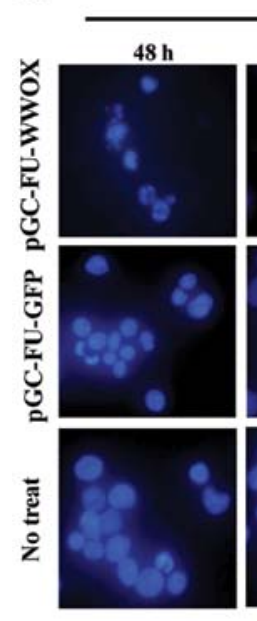

Jurkat

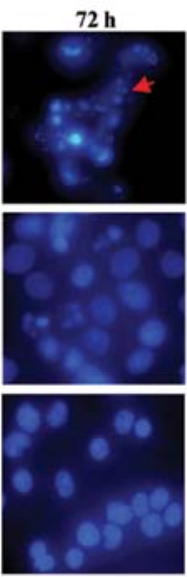

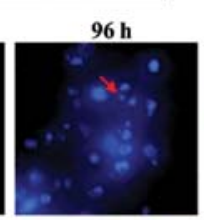
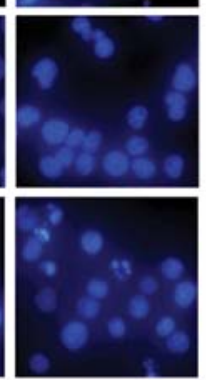

K562

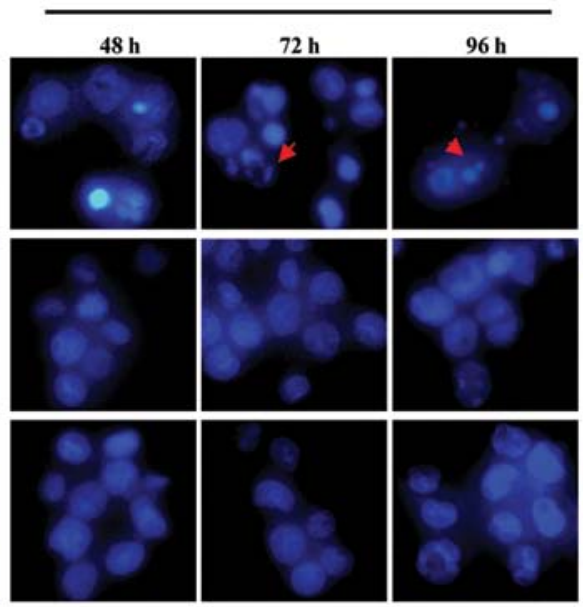

B

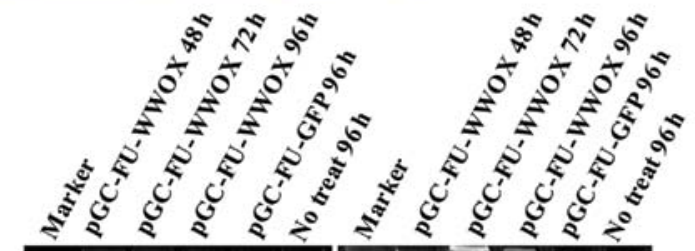

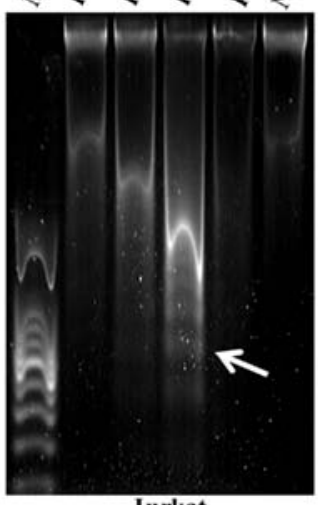

Jurkat

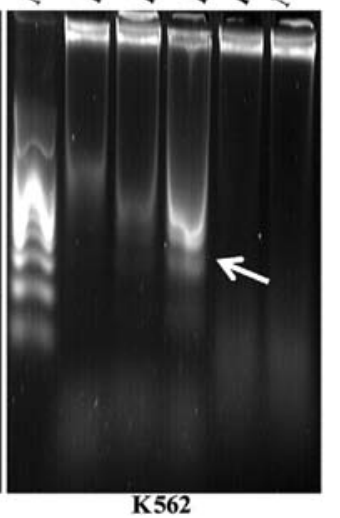

Figure 3. Wwox restoration promotes apoptosis in Jurkat and K562 cells. (A) DAPI fluorescence staining shows the microscopic changes of nucleus for lentivirus-infected cells at different time points observed by fluorescence microscopy, $\mathrm{x} 400$. Red arrows show a typical nucleus morphology of apoptosis in pGC-FU-WWOX-infected cells. (B) DNA fragmentation analysis using a DNA fragmentation kit. White arrows indicate the 'DNA ladders' in pGC-FUWWOX-infected cells with time lapse.

Wwox restoration promotes apoptosis in Jurkat and $K 562$ cells. As shown in Fig. 3A, there were apparent microscopic changes of nucleus in pGC-FU-WWOX-infected cells with a morphology of chromatin condensation and shrinkage in phase IIb of apoptosis exhibited by DAPI staining. DNA degradative fragments were detected by DNA ladder electrophoresis, which also showed typical increased apoptosis 'DNA ladders' in pGC-FU-WWOX-infected cells with time lapse when compared with the controls (Fig. 3B).

Bcl-2, Bax, caspase-3 and -9 are involved in WWOX-mediated apoptosis. To investigate whether WWOX restoration could induce apoptosis-related factors, we assessed the changes of Bcl-2, Bax, caspase-3 and -9 in both protein and mRNA levels. Real-time PCR results indicated that Bcl-2 mRNA in pGC-FU-WWOX-infected cells decreased, while Bax mRNA (only in K562 cells) increased with time lapse, all with $\mathrm{P}<0.05$ when vs. pGC-FU-GFP-infected cells (Fig. 4A). Western blot analysis revealed similar results for Bcl-2 and Bax. In addition, the Bax protein in Jurkat cells was undetectable although Bax
mRNA showed positively in it (Fig. 4A and B). Furthermore, western blot analysis displayed both procaspase-9 and -3 were cleaved by presenting their spliceosomes: $37 \mathrm{kDa}$ spliceosome for procaspase- 9 and $17 \mathrm{kDa}$ for procaspase-3 were observed in pGC-FU-WWOX-infected cells (Fig. 4B). Although the $17 \mathrm{kDa}$ spliceosome of procaspase-3 in pGC-FU-WWOXinfected Jurkat cells was not exposed, procaspase-3 decreased with time lapse (Fig. 4B).

Release of cytochrome $c$ is a result of WWOX-mediated apoptosis in Jurkat and K562 cells. To explore whether WWOX restoration could trigger the release of cytochrome $c$ from mitochondria, we employed immunofluorescence staining and western blot assay. Our results revealed that cytochrome $c$ for pGC-FU-WWOX-infected cells located in the cytosol with an increased, dispersive or block-like distribution (Fig. 5A). Moreover, total cytochrome $c$ protein as well as in the cytosol for pGC-FU-WWOX-infected cells increased and in mitochondria it decreased with time lapse, all with $\mathrm{P}<0.05$ when compared with pGC-FU-GFP-infected cells (Fig. 5B). 


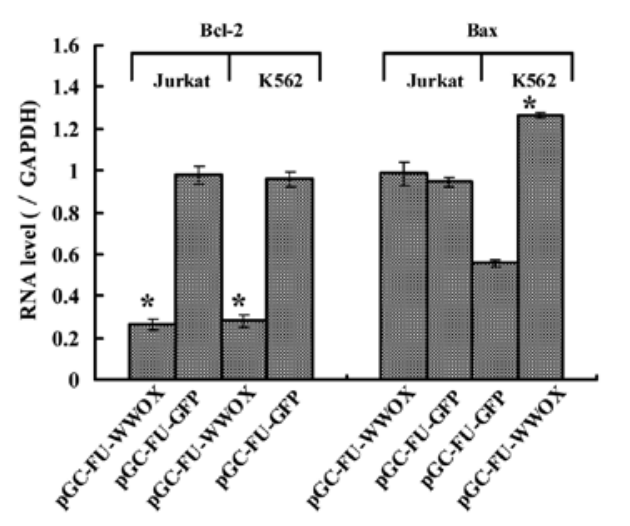

C

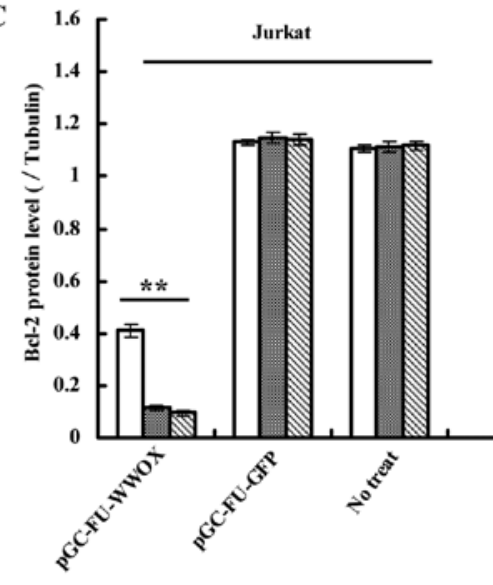

D

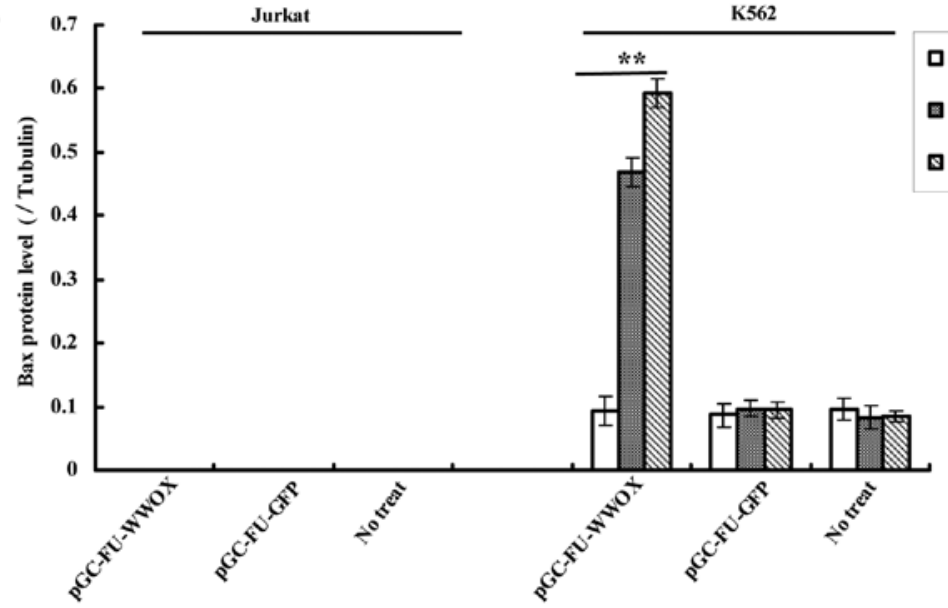

B pGC-FU-Wwox pGC-FU-GFP No treat
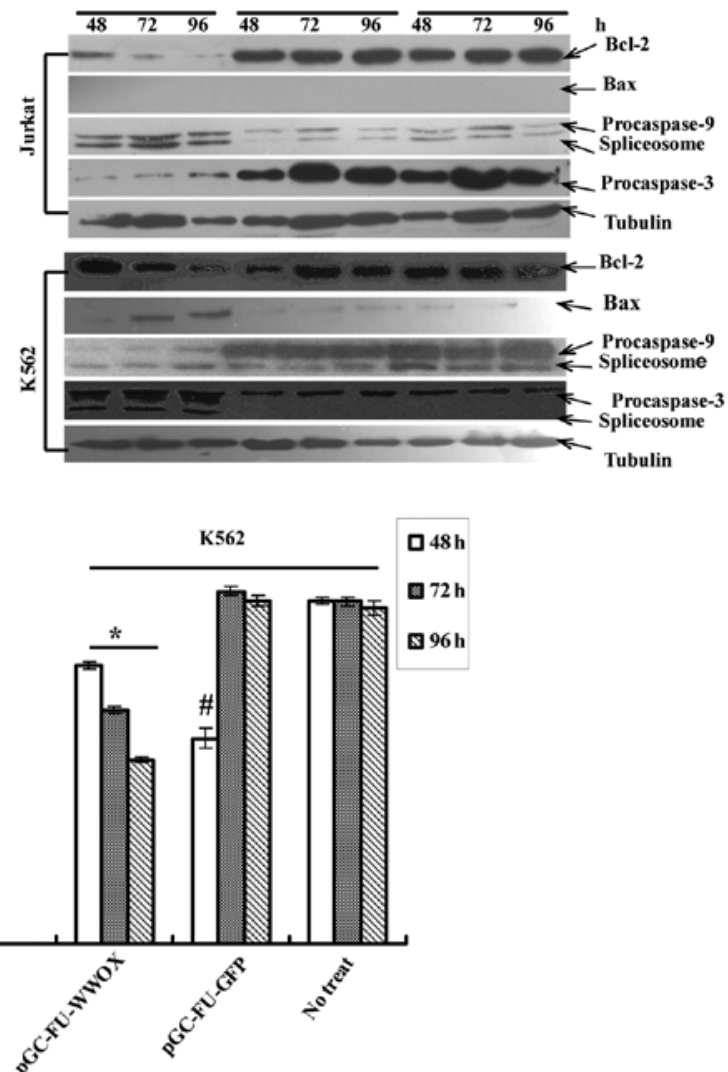

$72 \mathrm{~h}$

$96 \mathrm{~h}$
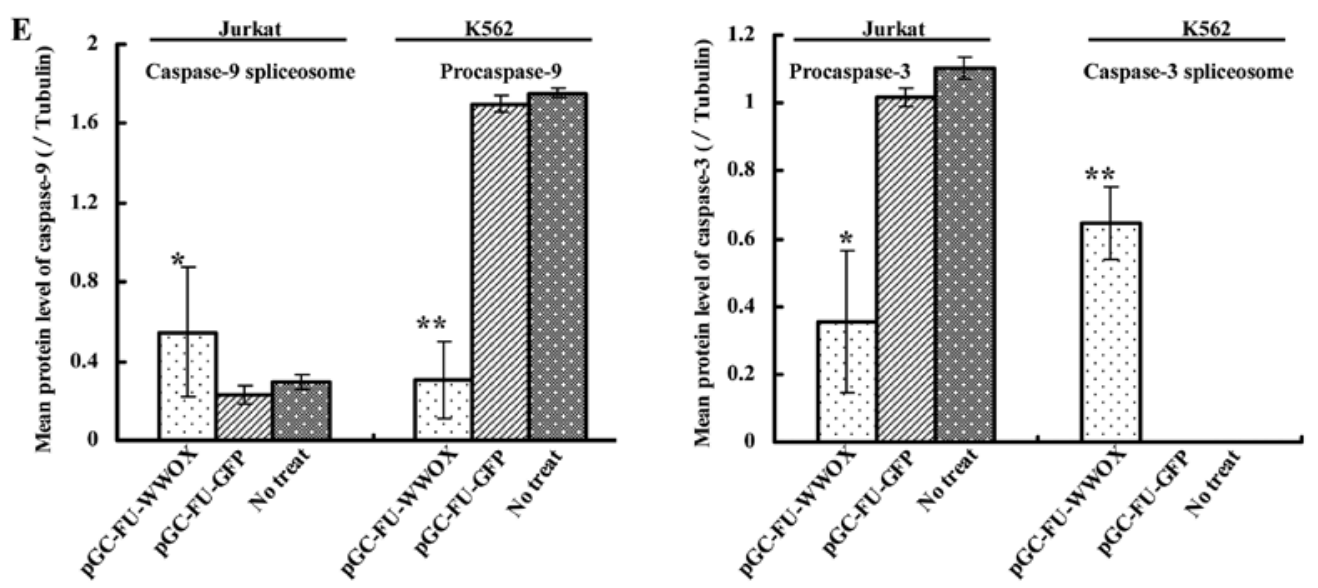

Figure 4. Expression changes of Bcl-2, Bax, caspase-3 and -9 induced by Wwox restoration. (A) Real-time PCR analysis. RNA was extracted at $72 \mathrm{~h}$ following infection, and the relative quantity was normalized to GAPDH. (B) Western blot analysis. Expression changes for (C) Bcl-2 protein, (D) Bax protein, (E) caspase-3 and -9 protein (mean expression level) at 48,72 and $96 \mathrm{~h}$ following infection were normalized to tubulin protein, and data shown are the means \pm SD $(\mathrm{n}=3$ or 6$) .{ }^{*} \mathrm{P}<0.05,{ }^{* *} \mathrm{P}<0.01$, vs. non-treated cells; ${ }^{*}$ unused value may be caused by measurement error. 
A

pGC-FU-WwOX

pGC-FU-GFP

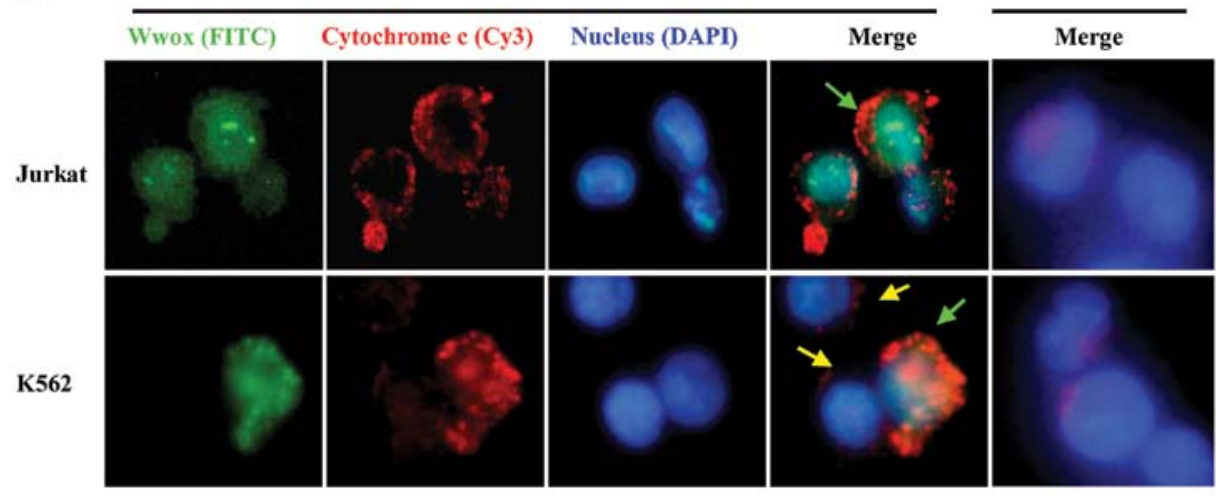

B $\quad$ pGC-FU-WWOX-infected Jurkat cells
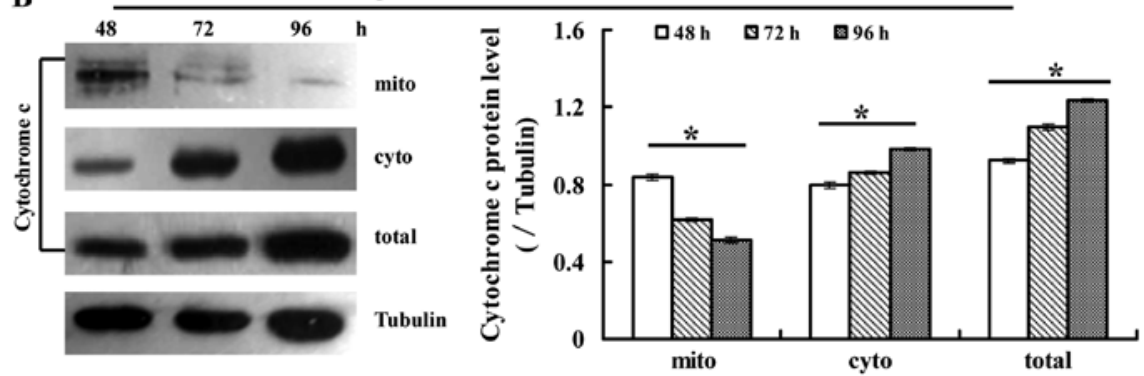

pGC-FU-GFP-infected Jurkat cells
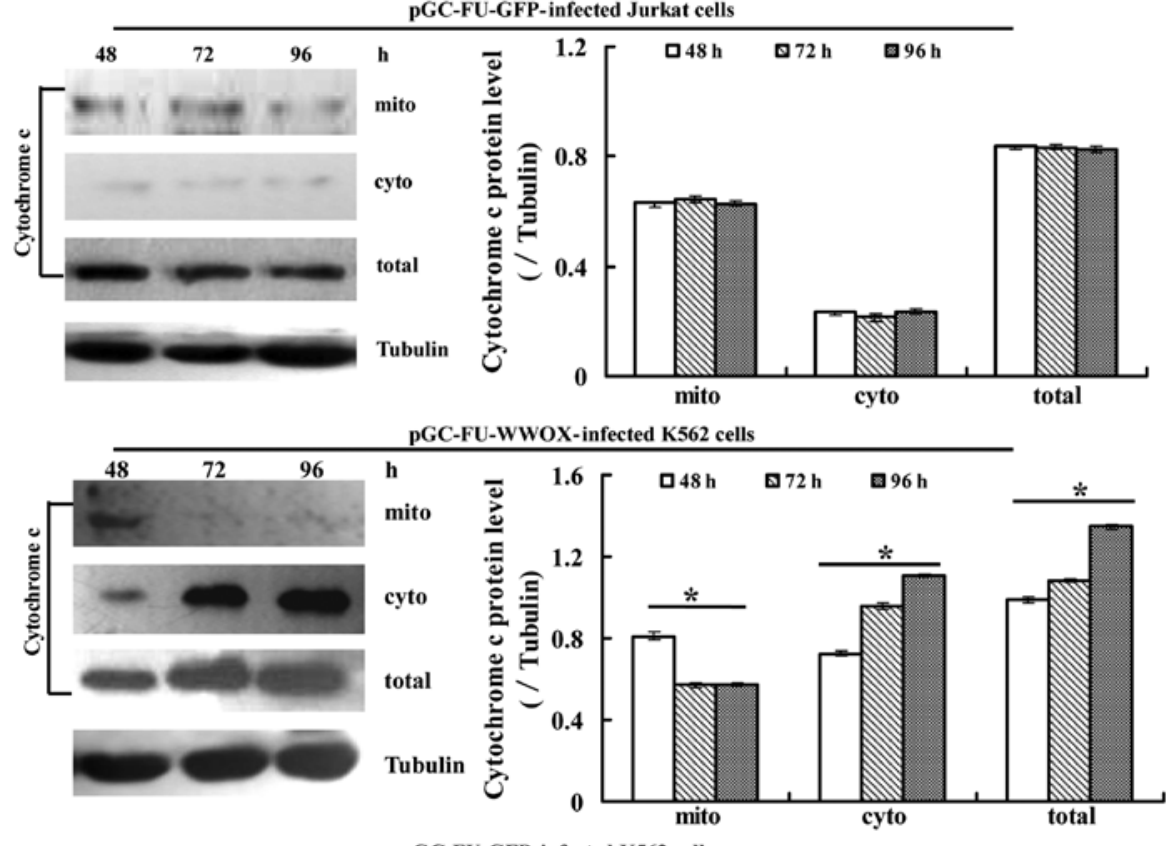

pGC-FU-GFP-infected K562 cells

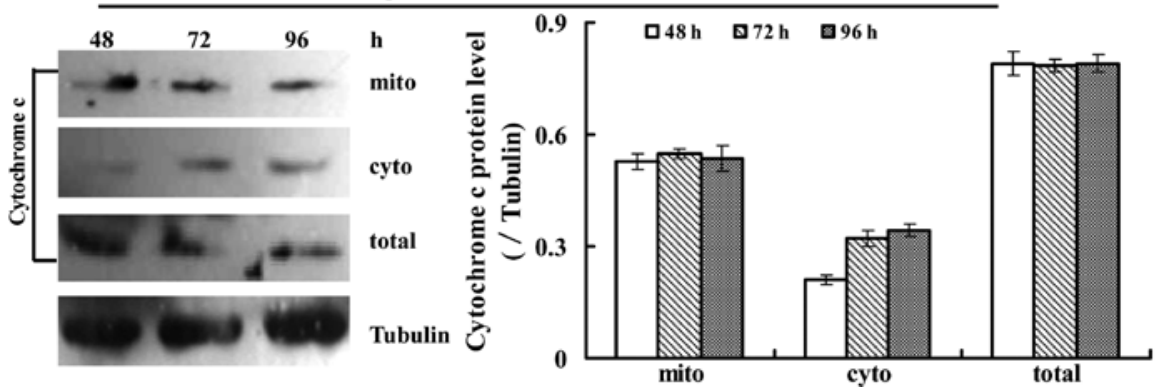

Figure 5. Release of cytochrome $c$ caused by Wwox restoration was measured by immunofluorescence staining assay and western blot analysis. (A) Immunofluorescence staining assay, observed by fluorescence microscopy at $60 \mathrm{~h}$ after infection, $\mathrm{x} 400$. As GFP could be easily destroyed by the denaturant while staining, green fluorescence FITC-conjugated anti-rabbit IgG was reapplied to remark Wwox, and cytochrome $c$ was marked by red fluorescence Cy3-conjugated anti-mouse IgG. The nucleus was stained by DAPI. Green arrows show the domain where Wwox and cytochrome $c$ merged in an orange patch in pGC-FU-WWOX-infected cells, and yellow arrows show the lentivirus-uninfected cells with no green fluorescence Wwox-GFP expression as well as low expression of cytochrome $c$. (B) Western blot analysis. Proteins from different organelles were extracted at 48, 72 and $96 \mathrm{~h}$ after infection, and normalized to tubulin. Data shown are the means $\pm \mathrm{SD}(\mathrm{n}=3)$. "P<0.05, vs. non-treated cells; cyto, cytosol; mito, mitochondria; total, total protein. 


\section{Discussion}

In the present study, we first evaluated the expression of WWOX in 38 cases of primary leukemia patients and in 10 leukemic cell lines, and observed a low expression level of WWOX in leukemia. Then, we restored Wwox protein expression in endogenous Wwox-negative cell lines Jurkat and K562 by utilizing the lentiviral vector pGC-FU-WWOX, and explored the effects of Wwox expression on biological properties of these cell lines. Our results exhibited that Wwox restoration resulted in significant cell viability inhibition and apoptosis in both Jurkat and K562 cells. We further investigated the possible mechanism underlying this process and whether WWOX regulates cell death via the mitochondrial pathway in leukemia. Finally, we found that restored Wwox promoted cytochrome $c$ release from the mitochondria and also activated caspase- 9 and -3 , indicating that WWOX could activate the mitochondrial pathway in its antitumor activities in leukemia.

Leukemia is an oligoclonal or monoclonal disease, and its molecular basis remains poorly understood. The WWOX gene was previously identified from chromosome region 16q23.324.1 which spans the common fragile site FRA16D (1), and low expression of WWOX has also been reported in various types of cancer (5-9). The present study also showed that expression of WWOX (mRNA and protein) was frequently reduced or lost in different types of primary leukemia patients and cell lines, but exhibited a high expression level in healthy volunteers. Recent evidence suggests that loss of heterozygosity, as well as epigenetic modification of the promoter by methylation, can reduce WWOX expression in various types of tumor $(7,23,24)$.

Apoptosis plays a key role in tumor viability or development, and a lack or failure of cell apoptosis in tumors leads to their development; thus, inducing cell apoptosis in tumors could be a candidate strategy for a new therapeutic approach for oncotherapy. Bednarek et al $(1,25)$ first reported that ectopic expression of WWOX could inhibit breast cancer viability both in vitro and in vivo. Gourley et al (26) revealed that WWOX expression abolishes ovarian cancer tumorigenicity in vivo, and Fabbri et al (10) also found an increase of WWOX in lung cancer cells exhibits marked suppression of tumorigenicity. Recent publications demonstrated that ectopic expression of WWOX leads to cell apoptosis in tumors such as lung cancer, glioblastoma multiforme, hepatoma, ovarian and prostate cancer as well $(17,19,24,27,28)$. Consistent with these findings, we also observed a restoration of Wwox in its absent leukemic cells led to a marked inhibition of cell growth and colony formation, and apoptosis effects were exhibited by the microscopic changes of nucleus in phase IIb of apoptosis accompanied by DNA ladders appearing in pGC-FU-WWOX-infected cells. Although there are opposing views regarding the function of WWOX as a tumor suppressor gene (29), the role of ectopic expression of WWOX in prohibiting proliferation and promoting apoptosis in leukemia is examined in this study.

WWOX is closely related to apoptosis-associated factors including Bcl-2, Bcl-xL and caspase in its antitumor activities (10,16-19), suggesting that WWOX may play a role in triggering apoptosis-associated pathways. Our study demonstrated that Wwox restoration could activate the mitochondrial pathway in Jurkat and K562 cells, as our results displayed ectopic expression of WWOX resulted in a promotion of cytochrome $c$ release from the mitochondria, even a downregulation of Bcl-2 and upregulation of Bax (only in K562 cells). Furthermore, both procaspase-3 and -9 were cleaved as their spliceosomes were detected in pGC-FU-WWOXinfected cells, although the $17 \mathrm{kDa}$ spliced caspase- 3 in Jurkat cells was difficult to detect, perhaps due to limitations in our experiment.

Supporting evidence of a possible association between WWOX and the mitochondrial pathway from Chang's review showed that overexpression of WWOX in L929 cells leads to an upregulation of the proapoptotic p53, as well as a downregulation of Bcl-2 and Bcl-xL (18). Fabbri et al (10) reported that restoration of WWOX in lung cancer cell lines enhances apoptosis by activating the intrinsic apoptotic caspase cascade. Zhang et al (17) recently transfected A549 cells with pcDNA3.0-WWOX, and found that the ectopic expression of WWOX not only caused apoptosis in A549 cells, but it also triggered caspase cascade, as well as a release of cytochrome $c$ from the mitochondria in A549 cells, indicating that the mitochondrial pathway is mainly involved in WWOX-mediated apoptosis in A549 cells. Hu et al (24) recently presented similar results. Our findings are consistent with these data.

In summary, this study reveals a functional role of WWOX in human hematopoietic malignancies as well as the molecular mechanisms of its proapoptotic activities. However, the number of cases examined in our study may not be sufficient, and we also did not evaluate the functional role of WWOX in leukemia in vivo. Clinical characterization of WWOX in hematopoietic malignancies warrants further study. Further investigations are also required to provide additional insights into the mechanism underlying the molecular action of WWOX in leukemia.

\section{Acknowledgements}

This study was supported by the Provincial Natural Science Fund of Fujian, grant no. 2010J01181.

\section{References}

1. Bednarek AK, Laflin KJ, Daniel RL, Liao Q, Hawkins KA and Aldaz CM: WWOX, a novel WW domain-containing protein mapping to human chromosome 16q23.3-24.1, a region frequently affected in breast cancer. Cancer Res 60: 2140-2145, 2000.

2. Ried K, Finnis M, Hobson L, et al: Common chromosomal fragile site FRA16D sequence: identification of the FOR gene spanning FRA16D and homozygous deletions and translocation breakpoints in cancer cells. Hum Mol Genet 9: 1651-1663, 2000.

3. Del Mare S, Salah Z and Aqeilan RI: WWOX: Its genomics, partners, and functions. J Cell Biochem 108: 737-745, 2009.

4. Macias MJ, Wiesner S and Sudol M: WW and SH3 domains, two different scaffolds to recognize proline-rich ligands. FEBS Lett 513: 30-37, 2002

5. Wang X, Chao L, Jin G, Ma G, Zang Y and Sun J: Association between $\mathrm{CpG}$ island methylation of the WWOX gene and its expression in breast cancers. Tumour Biol 30: 8-14, 2009.

6. Guo W, Wang G, Dong Y, Guo Y, Kuang G and Dong Z: Decreased expression of WWOX in the development of esophageal squamous cell carcinoma. Mol Carcinog: Dec 27, 2011 (Epub ahead of print). doi: 10.1002/mc.21853.

7. Nakayama S, Semba S, Maeda N, Matsushita M, Kuroda Y and Yokozaki H: Hypermethylation-mediated reduction of WWOX expression in intraductal papillary mucinous neoplasms of the pancreas. Br J Cancer 100: 1438-1443, 2009. 
8. Dias EP, Pimenta FJ, Sarquis MS, et al: Association between decreased WWOX protein expression and thyroid cancer development. Thyroid 17: 1055-1059, 2007.

9. Yang J, Cogdell D, Yang D, et al: Deletion of the WWOX gene and frequent loss of its protein expression in human osteosarcoma. Cancer Lett 291: 31-38, 2010.

10. Fabbri M, Iliopoulos D, Trapasso F, et al: WWOX gene restoration prevents lung cancer growth in vitro and in vivo. Proc Natl Acad Sci USA 102: 15611-15616, 2005.

11. Iliopoulos D, Fabbri M, Druck T, Qin HR, Han SY and Huebner K: Inhibition of breast cancer cell growth in vitro and in vivo: effect of restoration of Wwox expression. Clin Cancer Res 13: 268-274, 2007.

12. Aqeilan RI, Hagan JP, Aqeilan HA, Pichiorri F, Fong Ly and Croce CM: Inactivation of the WWOX Gene accelerates forestomach tumor progression in vivo. Cancer Res 67: 5606-5610, 2007.

13. Aqeilan RI, Trapasso F, Hussain S, et al: Targeted deletion of Wwox reveals a tumor suppressor function. Proc Natl Acad Sci USA 104: 3949-3954, 2007.

14. Ishii H, Vecchione A, Furukawa Y, et al: Expression of FRA16D/ WWOX and FRA3B/FHIT genes in hematopoietic malignancies. Mol Cancer Res 1: 940-947, 2003.

15. Ishii $\mathrm{H}$ and Furukawa Y: Alterations of common chromosome fragile sites in hematopoietic malignancies. Int J Hematol 79: 238-242, 2004

16. Yang JL and Zhang W: WWOX tumor suppressor gene. Histol Histopathol 23: 877-882, 2008

17. Zhang P, Jia R, Ying L, Liu B, Qian G, Fan X and Ge S: WWOX-mediated apoptosis in A549 cells mainly involves the mitochondrial pathway. Mol Med Rep 6: 121-124, 2012.

18. Chang NS: A potential role of $\mathrm{p} 53$ and WOX1 in mitochondrial apoptosis (review). Int J Mol Med 9: 19-24, 2002.

19. Kosla K, Pluciennik E, Kurzyk A, Jesionek-Kupnicka D, Kordek R, Potemski P and Bednarek AK: Molecular analysis of WWOX expression correlation with proliferation and apoptosis in glioblastoma multiforme. J Neurooncol 101: 207-213, 2011.
20. Zhou YL, Xu YJ and Zhang ZX: A study on expression changes of a tumor suppressor WWOX in human lung adenocarcinoma cell line A549. China Oncology 15: 234-237, 2005.

21. Wang XF and He YL: Construction of a lentiviral vector carrying human bcl-2 gene and its expression in human ovarian granulosa cells. Nan Fang Yi Ke Da Xue Xue Bao 28: 1856-1859, 2008 (In Chinese).

22. Reagan-Shaw S, Nihal M, Ahsan H, Mukhtar H and Ahmad N Combination of vitamin $\mathrm{E}$ and selenium causes an induction of apoptosis of human prostate cancer cells by enhancing $\mathrm{Bax} / \mathrm{Bcl}-2$ ratio. Prostate 68: 1624-1634, 2008.

23. Iliopoulos D, Guler G, Han SY, et al: Fragile genes as biomarkers: epigenetic control of WWOX and FHIT in lung, breast and bladder cancer. Oncogene 24: 1625-1633, 2005.

24. Hu BS, Tan JW, Zhu GH, Wang DF, Zhou X and Sun ZQ: WWOX induces apoptosis and inhibits proliferation of human hepatoma cell line SMMC-7721. World J Gastroenterol 18: 3020-3026, 2012.

25. Bednarek AK, Keck-Waggoner CL, Daniel RL, et al: WWOX, the FRA16D gene, behaves as a suppressor of tumor growth. Cancer Res 61: 8068-8073, 2001.

26. Gourley C, Paige AJ, Taylor KJ, et al: WWOX gene expression abolishes ovarian cancer tumorigenicity in vivo and decreases attachment to fibronectin via integrin alpha3. Cancer Res 69: 4835-4842, 2009.

27. Xiong Z, Hu S and Wang Z: Cloning of WWOX gene and its growth-inhibiting effects on ovarian cancer cells. J Huazhong Univ Sci Technolog Med Sci 30: 365-369, 2010.

28. Qin HR, Iliopoulos D, Semba S, et al: A Role for the WWOX gene in prostate cancer. Cancer Res 66: 6477-6480, 2006

29. Watanabe A, Hippo Y, Taniguchi H, et al: An opposing view on WWOX protein function as a tumor suppressor. Cancer Res 63: 8629-8633, 2003. 